Décadrages Décadrages

cinéma, à travers champs Cinéma, à travers champs

$7 \mid 2006$

Stephen Dwoskin

\title{
Snow White : tout blanc tout noir
}

\section{Alain Boillat}

\section{(2) OpenEdition}

Journals

Édition électronique

URL : https://journals.openedition.org/decadrages/470

DOI : $10.4000 /$ decadrages. 470

ISSN : 2297-5977

\section{Éditeur}

Association Décadrages

\section{Édition imprimée}

Date de publication : 10 avril 2006

Pagination : 110-112

ISBN : 978-29700582-3-6

ISSN : 2235-7823

Référence électronique

Alain Boillat, «Snow White : tout blanc tout noir », Décadrages [En ligne], 7 | 2006, mis en ligne le 30 janvier 2014, consulté le 03 avril 2022. URL : http://journals.openedition.org/decadrages/470 ; DOI https://doi.org/10.4000/decadrages.470

Ce document a été généré automatiquement le 3 avril 2022.

(B) Décadrages 


\title{
Snow White : tout blanc tout noir
}

\author{
Alain Boillat
}

1 Le dernier long métrage de fiction réalisé par Samir, cinéaste d'origine irakienne établi à Zurich, nous conte les rebondissements de la relation passionnée que nouent Nico (Julie Fournier), fille à papa nageant dans l'opulence, la luxure et la coke, et Paco (Carlos Leal) ${ }^{1}$, chanteur de rap français issu d'une banlieue. L'opposition sociale constitue certes un topos éculé, mais l'attention portée d'un point de vue sociologique à l'effondrement progressif et inéluctable de la jeune femme en dépit de ses efforts pour changer de vie pimente l'évolution des relations du couple, jusqu'à une renaissance où la blancheur de la poudre sniffée s'étend à l'ensemble du paysage, un finale marqué par la sérénité et la pureté. «Dure est la chute », tel est cependant l'aphorisme que le film s'évertue ouvertement à signifier en faisant croire qu'il se débarrasse de toute candeur dans sa peinture (en fait passablement caricaturale) de l'environnement de la riche Zurichoise ${ }^{2}$.

2 On ressent une gêne quelque peu «problématique " (c'est-à-dire intéressante du fait même qu'elle fasse problème) dans le portrait du personnage féminin qui tient à un détail technique, mais innerve à mon sens l'entièreté du film au niveau du point de vue qu'il adopte. En effet, la voix de Nico a été ostensiblement post-synchronisée, puisqu'elle n'appartient pas à l'actrice visible à l'écran, mais semble flotter sur ses lèvres lorsque celle-ci est filmée en gros plan. Toute voix ainsi « déliée » n'est bien sûr pas à bannir, au contraire : il réside dans cette interaction entre le son et l'image un potentiel esthétique certain. Toutefois, ce léger décalage n'a de l'intérêt que s'il est travaillé en tant que tel, par exemple dans le sens d'une étrangéification de la représentation. Snow White fonctionne par contre sur un rapport direct aux acteurs qui s'accommode mal de la déliaison, si ce n'est au cours du « chemin de croix » de Nico lorsque, devenue fantomatique, elle n'en finit pas de s'évanouir dans l'image au gré d'une série de fondus. On arguera que le synchronisme dépend de la "subjectivité » du spectateur ${ }^{3}$, et se justifie entièrement par le contexte plurilinguistique suisse (il eût fallu une actrice bilingue, ce qui n'est pas évident) que le film exploite partiellement et dont Samir traite de façon approfondie dans un documentaire également réalisé en 2005 et présenté à Soleure, ZwischenSprach ${ }^{4}$. La déliaison vocale acquiert néanmoins dans Snow White une dimension particulière dans la mesure où les deux personnages 
s'expriment aussi over pour commenter les actions ou introduire de brefs flash-backs. Or le film débute avec la voix-over de Paco qui s'ancre subrepticement in, en toute continuité, affirmant l'emprise physique du personnage sur les énoncés verbaux qui organisent le film à partir de la position de surplomb que lui confère le statut over. Par contre, en ce qui concerne Nico, la voix demeure over lorsque l'actrice apparait, ce qui permet certes d'éviter momentanément tout problème de synchronisation, mais introduit une différence notable de traitement qui est à mon sens révélatrice d'une disjonction plus profonde : l'autorité de la parole, et par là l'affirmation d'un système de valeurs, passent majoritairement par le personnage masculin, dont la figure de « rebelle» est érigée en modèle par le film. La voix plaquée sur l'actrice ne fait par exemple pas « le poids » - celui attribué aux dires par la corporéité d'un locuteur - lors de la confrontation entre les amants qui a lieu dans la somptueuse villa de la Goldküste. Alors que la jeune femme tente de convaincre Paco de nuancer sa vision manichéenne, le film lui-même donne unilatéralement raison à ce dernier, cristallisant in fine cette valorisation dans l'affirmation du père, immigré espagnol, disant à son fils qu'il « est quelqu'un de bien ». Significativement, c'est après une tentative de suicide où Nico se blesse à l'aire de Broca (centre des capacités langagières) que les retrouvailles s'avèreront possibles: autrement dit, une fois la femme privée de la capacité de s'exprimer verbalement.

3 Cette façon de véhiculer des valeurs à travers la figuration du personnage masculin, individu fidèle à ses principes, libre et décidé, exige certaines ficelles scénaristiques parfois fort dispensables 5 . Il y a effectivement dans Snow White une volonté par trop évidente d'" approfondir » la psychologie de Paco. Ainsi le cinéaste a-t-il par exemple cru bon de mâtiner superficiellement le caractère de son personnage des affres de la culpabilité, le frère de Paco étant mort d'une overdose (heureux hasard pour la «morale» du film). Par conséquent, le travail convaincant au niveau du montage (y compris à l'intérieur du plan, qui connaît divers types de fragmentation) ne suffit pas toujours à faire oublier l'inanité des flash-backs consacrés au cadre familial de la famille du raper.

Globalement, l'esthétique du film de Samir se caractérise par un effet "clip vidéo » assumé, voire thématisé lorsque des images d'une party autour d'une piscine sont montrées une seconde fois en obéissant cette fois à une posture réflexive ${ }^{6}$. La juxtaposition de cadres de grandeur variable dynamise la lecture de l'image et du récit, mais la fragmentation spatiale et l'exacerbation du gros plan qu'elle entraîne enraie parfois l'inscription des personnages dans leur environnement. Par contre, le lieu bétonné de leur première étreinte nous est présenté, grâce à un brusque mouvement d'appareil (dont le statut oscille entre le tabou et la métonymie), comme le centre d'un réseau d'autoroutes. Ce plan souligne combien le film est ancré dans un contexte urbain (et cela dès le "panorama " de son plan liminaire), démarche peu fréquente chez les cinéastes helvétiques lorsqu'ils tournent en Suisse ${ }^{7}$. Pour cette raison, ce sont probablement les déambulations et faux pas de Nico et de son amie (une prostituée de luxe) dans la ville nocturne qui occasionnent les meilleurs moments du film.

Snow White (2005). Réal. : Samir. Scénario : M. Sauter et Samir. Image : A. Hutter, M. Saxer, H. Meier. Montage : O. Neumann. Son : Dieter Meyer. Interprétation : J. Fournier, C. Leal, Production : Dschoint Ventschr Filmproduktion AG (Zurich) / Filmhaus Films (Vienne). 


\section{NOTES}

1. Le chanteur du groupe hip hop Sens Unik (dissout en 2005), déjà présent dans un film antérieur de Samir (le documentaire Babylon 2, 2003), a reçu pour son rôle dans Snow White le prix du meilleur acteur principal aux Journées de Soleure.

2. Alors qu'on nous fait explicitement comprendre, dans des dialogues où ces films sont mentionnés avec cynisme, que nous ne sommes ni dans Pretty Woman ni dans Evita, la représentation « onirique » du coup de foudre entre le chanteur sur scène et la jeune femme en lévitation dans le public semble ne pas craindre ce type de ridicule.

3. Sur le spectateur pèsent en effet des déterminations sociales (tout le monde ne regarde pas systématiquement des versions originales) et culturelles. Ces dernières peuvent être également définies par des pratiques dominantes à un niveau national. Michel Chion fait par exemple remarquer une différence notoire entre la France et l'Italie (L'Audio-vision, Paris, Nathan, p. 57), les habitants de la Péninsule ayant été habitués (peut-être par le biais des nombreuses productions internationales des années 1970) à plus de tolérance envers le degré de précision du synchronisme vocolabial. En Suisse par contre, la diversité des zones linguistiques favorise plutôt la distribution de copies sous-titrées.

4. En dépit des différences radicales qui distinguent un film de reportage et une fiction, on note plusieurs parentés formelles entre Snow White et ZwischenSprach, notamment au niveau de l'utilisation des splits screens multiples et du graphisme des mentions écrites.

5. Il ne s'agit pas tant d'invraisemblance (certains événements revêtent au contraire une valeur biographique par rapport à la vie de Carlos Leal) que du mode et du lieu de l'intervention de ces éléments de récit dans l'économie narrative générale du film.

6. L'apparition du clap dans le plan nous révèle qu'il s'agit d'images tournées pour un clip musical que le groupe de Paco est en train de réaliser.

7. Inversement, Samir ne montre presque rien d'autre de Paris - ville au lourd passé cinématographique - que l'intérieur de l'appartement acheté par Paco. 